\title{
Logarithmic Corrections for Spin Glasses, Percolation and Lee-Yang Singularities in Six Dimensions
}

\author{
Juan J. Ruiz-Lorenzo \\ Departamento de Física Teórica I \\ Facultad de Ciencias Físicas \\ Universidad Complutense de Madrid \\ Ciudad Universitaria \\ 28040 Madrid (Spain) \\ ruiz@lattice.fis.ucm.es
}

June 26, 1998

\begin{abstract}
We study analytically the logarithmic corrections to the critical exponents of the critical behavior of correlation length, susceptibility and specific heat for the temperature and the finite-size scaling behavior, for a generic $\phi^{3}$ theory at its upper critical dimension (six). We have also computed the leading correction to scaling as a function of the lattice size. We distinguish the obtained formulas to the following special cases: percolation, Lee-Yang (LY) singularities and $m$-component spin glasses. We have compared our results for the Ising spin glass case with numerical simulations finding a very good agreement. Finally, and using the results obtained for the Lee-Yang singularities in six dimensions, we have computed the logarithmic corrections to the singular part of the free energy for lattice animals in eight dimensions.
\end{abstract}




\section{Introduction}

One of the techniques commonly used in the study of statistical systems is to perform numerical simulations focusing on finite size effects. The main tool of this approach is the knowledge of how some observables diverge in the critical region as a function of the size of the system instead of the usual formulas that express these divergences as a function of the reduced temperature (or in reduced probability in the case of percolation) or magnetic field. Moreover it is possible to measure these finite size effects in experiments.

For statistical systems below their upper critical dimensions there is an extensive literature on this subject [1].

The main goal of this work is to obtain the functional form of the divergences, as functions of the reduced temperature as well as the lattice size. We focus on observables commonly measured in numerical simulations at the upper critical dimension, for a wide class of systems like the vector spin glasses [2], percolation [3] and Lee-Yang [4, 34] (LY) singularities. A classical feature of the upper critical dimension is that the critical behavior (which is described by the critical exponents) is modified by logarithms. The logarithmic corrections to the critical behavior of the susceptibility (spin glasses (in reduced temperature) and percolation (in probability)) and correlation length (for percolation, in probability) were computed in references [21] (percolation) and [23] (spin glasses). We will use these previous results as check of our calculation.

Moreover, and using the mapping proposed by Parisi and Sourlas [33] between the actions which describe the Lee-Yang singularities for the Ising model in $d$ dimensions and the lattice animals in $d+2$ dimensions, we have been able to compute the logarithmic correction to the singular part of the free energy for lattice animals [3, 35] at its upper critical dimension (eight). To check the mapping (a further check) we have compared our result for the LY singularities with that of reference [31] where originally was computed the logarithmic correction to the free energy directly for lattice animals and with reference [32 where it was checked using series expansions.

The understanding of these logarithms has very important physical applications. For instance, the $\phi^{4}$ theory in four dimensions (that we denote as $\phi_{4}^{4}$ ) is trivial (the theory that we obtain when the cut-off is sent to infinity is a free, non-interacting, theory) because the logarithmic corrections produce a vanishing renormalized coupling constant \$.

Another example where the knowledge of the logarithmic factors is very important is the study of the uniaxial system with strong dipolar forces. In this system the upper critical dimension is just three and thereby, the theoretical predictions for the logarithmic corrections have been checked experimentally, the agreement being very good [9].

In this note we mainly focus on the study of the logarithms in the field theory description of spin glasses, a $\phi^{3}$ theory 2 The generic $\phi^{3}$ theory (i.e. the coupling is a generic tensor $\left.\lambda_{i j k}\right)$ also describes a large set of interesting statistical systems. We can cite, for instance, the $q$-states Potts model, percolation and Lee-Yang singularities (described by one-component $\phi^{3}$

\footnotetext{
${ }^{1}$ At the critical point, the renormalized constant, $g_{R}=(L / \xi)^{d} B$ (where $\xi$ is the correlation length, $L$ is the lattice size, $d$ is the dimension and $B$ is the Binder cumulant), of the four dimensional Ising model drops following a law: $g_{R} \propto 1 / \log L[16]$.

2 This theoretical description only holds in the paramagnetic phase. To study the spin glass phase we need to consider a $\phi^{4}$ term that induces the breaking of the replica symmetry in infinite dimensions [5].
} 
theory with a purely imaginary coupling [34]).

The study of spin glasses in finite dimensions is another interesting current research issue [5]. It is very important to understand if the strange and interesting properties of the Parisi solution (which is believed to be exact in infinite dimensions) apply in finite dimensions [5]. In particular, the existence of a large number (infinite) of pure states which organize in an ultrametric fashion is an open problem in the current spin glass research.

There exist two analytical approaches that try to answer these questions: The first one is the droplet model [14, 15] that predicts that the spin glass phase is composed by one pure state (and its inverse by flipping all spins). The underlying approximation is the MigdalKadanoff one that is an approximate real space renormalization group. The Migdal-Kadanoff technique is known to give exact answers in one dimension and also that it lacks of predictive power when the dimensionality grows. For instance, the Migdal-Kadanoff approach is unable to predict the Mean Field exponents of the four dimensional (ordered) Ising model.

The second method is based on the Mean Field approximation. This approach is the classical one that has worked fine in the ordered Ising Model. Firstly one solves the model in infinite dimensions, then it is possible to show that the critical properties of the system remain unchanged up to the so-called upper critical dimension (where the critical laws are modified by logarithms). Below the upper critical dimension the thermal fluctuations change the critical behavior, which can be analyzed using renormalization group techniques. This approach predicted that the upper critical dimension for spin glasses is six.

Our main goal is to calculate the logarithmic corrections that predicts the last approach (i.e. continuous formulation of the problem plus renormalization group) in six dimensions for the spin glass (its upper critical dimension) and to compare them with the logarithms found by Wang and Young [26] simulating the six dimensional spin glass.

Wang and Young performed extensive numerical simulations [26] in order to check whether six was really the upper critical dimension of the theory [17]. They found the Mean Field critical exponents $(\gamma=1$ and $\nu=1 / 2)$ but they also found logarithmic corrections, for example, looking at the finite size effects on the non linear susceptibility. Obviously to close this still open problem (i.e. whether six is really the upper critical dimension) it is mandatory to known whether the logarithmic corrections found by Wang and Young are those predicted by the theory.

Another point of interest is to check that at least when $\epsilon=0$ the approach has a predictive power. The convergence of the $\epsilon$-expansion is really poor for the $\phi^{3}$ theory (see [27] for an example of this poor convergence in percolation). In particular for a one component spin glass it is impossible to re-sum (in the Borel sense) the series for the critical exponents because all the known terms of the series has the same sign. This is why the Field Theory approach has not had a great success. But in this note we show that the underlying approach is indeed right: it predicts the right logarithmic corrections that has been found with the computer.

We calculate analytically in the present paper the logarithmic corrections, and we compare them with those seen by Wang and Young, finding a very good agreement.

The calculation of the logarithmic corrections (for the correlation length, the non linear susceptibility and the specific heat) has been done using two different analytical starting points:

1. The renormalization group recursion formulas, found by Harris, Lubensky and Chen 20] 
in the framework of the Wilson renormalization group [6]. In this case we have obtained the logarithmic corrections for the $m$-component spin glass.

2. The results of De Alcantara et al. 13, 18], obtained using a Field Theory approach [7]. In this case the coefficients, tensor, of the $\phi^{3}$ term in the actions are completely general and so we can distinguish our final formula to the following cases: percolation, Lee-Yang singularities and $m$-component spin glass (in this case using the results of [19]) P.

Of course, at the end we will get two predictions (but not fully independent) for the logarithmic corrections for the spin glass case, that agree between them: thus we have checked that the final formulas are right (for the spin glass at least). Moreover we have extended the computation to two other systems namely the percolation and the Lee-Yang singularities.

Another check of our calculation was done by comparing the logarithmic correction of the mean cluster size (a susceptibility) and the logarithm of the correlation length (in $p-p_{c}$ ) that we have found in percolation with the results of the reference [21, where they were computed using a Field Theory approach, the agreement being perfect. Moreover we have compared the logarithmic corrections to the critical behavior of the non linear susceptibility for the six dimensional spin glass with the results of reference [23] obtaining, again, the same formula for the non linear susceptibility.

We remark that we have extended the computation of the logarithmic corrections for percolation and spin glasses to another set of observables and, what is the main issue of the paper, we have computed the cited correction as a function of lattice size. The study of the six dimensional percolation and the six dimensional Ising spin glass using series expansions can be seen in references [22] and 24 respectively.

The plan of the paper is the following. In the next section we write down the analytical setup that we need in the rest of the paper: the renormalization group recursions of Harris et al., the Field Theory results of De Alcantara et al. and some useful Mean Field results. In sections 3 and 4 we deduce using the Wilson Renormalization Group (WRG) the logarithmic scaling correction as a function of temperature and lattice size respectively for the $m$-component Ising spin glass. In section 5 we generalize the previous results to percolation and Lee-Yang singularities (in temperature and lattice size for a $\phi^{3}$ theory with imaginary coupling) using the mapping between the results of Field Theory (FT) and WRG checking that for the Ising spin glass we recover the results of sections 3 and 4 . In section 6 we have computed the singular part of the free energy for a $\phi^{3}$ theory with imaginary coupling just at criticality and in presence of a magnetic field (LY singularities [34) and we have compared this result with that of lattice animals in eight dimension (in this case as a function of the fugacity, that plays the role of the magnetic field in LY singularities) obtaining the same result, a further test that the mapping (a perturbative mapping) suggested by Parisi and Sourlas [33] works even in presence of logarithms. Finally in section 7 we present the conclusions.

${ }^{3}$ Obviously in these works [13, 18, 12] there is information about the Potts model, but in six dimensions the Potts Model (with more than two states) shows a first order phase transition. 


\section{Analytical set-up}

In this section we will write the recursion formulas for the $m$-component spin glass found by Harris et al. [20] using renormalization group à la Wilson and the Field Theoretical renormalization group formulas obtained by De Alcantara et al. [13, 18].

Moreover we will write down some useful formulas in the Mean Field framework.

\subsection{Wilson renormalization group (WRG) equations}

One can obtain with the replica trick and assuming that the replica symmetry has not been broken, the following starting action for the $m$-component spin glass

$$
S=\int \mathrm{d}^{d} x\left[\frac{1}{2}\left(\partial_{i} \phi\right)^{2}+\frac{1}{4} m r \phi^{2}-w(n-2) \phi^{3}\right] .
$$

where $n$ is the number of replicas.

Harris, Lubensky and Chen [20] found in a renormalization group calculation à la Wil-

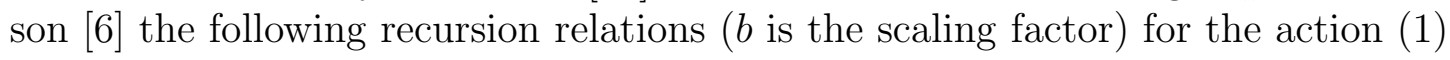

$$
\begin{aligned}
r^{\prime} & =b^{2-\gamma}\left(r-36(n-2) m w^{2}\left[A(0)-2 K_{6} r\right] \log b\right), \\
w^{\prime} & =b^{\epsilon / 2-3 \gamma / 2}\left(w+36[(n-3) m+1] w^{3} K_{6} \log b\right),
\end{aligned}
$$

with $\gamma=\gamma(w)=12(n-2) m w^{2} K_{6}$, and $\epsilon=6-d . A(0)$ and $K_{6}$ are constants.

In the spin glass case we assume we take the replica trick limit $(n \rightarrow 0)$ and the number of dimensions to be $\operatorname{six}(\epsilon=0)$.

We can write Eqs. (2) in a differential form, by performing a differential dilatation, obtaining

$$
\begin{aligned}
& \frac{\mathrm{d} r}{\mathrm{~d} \log b}=\left(2-120 K_{6} m w^{2}\right) r+72 m w^{2} A(0), \\
& \frac{\mathrm{d} w}{\mathrm{~d} \log b}=-36(2 m-1) K_{6} w^{3} .
\end{aligned}
$$

We denote $\beta_{\mathrm{W}}(w) \equiv \mathrm{d} w / \mathrm{d} \log b$.

Defining $t \equiv r+36 A(0) m w^{2}$ we recast the first equation of (国) in the standard form

$$
\frac{\mathrm{d} t}{\mathrm{~d} \log b}=\left(2-120 K_{6} m w^{2}\right) t .
$$

The solutions of the WRG equations (Eqs. (3) are

$$
\begin{aligned}
\log \frac{t(b)}{t_{0}} & =2 \log b-\frac{5 m}{3(2 m-1)} \log \left(1+72 w^{2}(1) K_{6}(2 m-1) \log b\right) \\
w^{2}(b) & =\frac{w^{2}(1)}{1+72 w^{2}(1) K_{6}(2 m-1) \log b}
\end{aligned}
$$


where $t_{0} \equiv t(b=1)$. We are interested in the asymptotic behavior that reads

$$
\begin{aligned}
t(b) & \sim t_{0} b^{2}(\log b)^{-5 m /(3(2 m-1))} \\
w(b) & \sim \frac{1}{\sqrt{72(2 m-1) K_{6}}}(\log b)^{-1 / 2} .
\end{aligned}
$$

In order to link the previous formulas with the Field Theory approach we recall

$$
\gamma(w)=-24 m w^{2} K_{6}
$$

and define $\bar{\gamma}$ as

$$
\frac{\mathrm{d} t}{\mathrm{~d} \log b}=t(2+\bar{\gamma}(w))
$$

obtaining

$$
\bar{\gamma}=-120 K_{6} m w^{2}
$$

Finally we can write, in this approach, the expression of the critical exponents as a function of $\eta(w)$ and $\bar{\gamma}(w)$ at the fixed point $w^{*}$ (where $\beta_{\mathrm{W}}\left(w^{*}\right)=0$ )

$$
\begin{aligned}
\nu & =\frac{1}{2+\bar{\gamma}\left(w^{*}\right)}, \\
\eta & =\gamma\left(w^{*}\right) .
\end{aligned}
$$

\subsection{Field Theory Formulas}

Taking the limit, $\epsilon \rightarrow 0$ in the formulas of references [13, 18] it is possible to write in the notation of Amit's book [7] 团

$$
\begin{aligned}
\beta(w) & =\left(\frac{1}{4} \alpha-\beta\right) w^{3}, \\
\gamma_{\phi}(w) & =\frac{1}{6} \alpha w^{2} \\
\bar{\gamma}_{\phi^{2}}(w) & =-\alpha w^{2} \\
\gamma_{\phi^{2}} & \equiv \bar{\gamma}_{\phi^{2}}+\gamma_{\phi}=-\frac{5}{6} \alpha w^{2} .
\end{aligned}
$$

The values for $\alpha$ and $\beta$ for different models are written in Table (11). We have taken the $\alpha$ and $\beta$ values from references 13, 18] (percolation and Lee-Yang singularities) and [19] (spin glasses).

Using the spin glass values for $\alpha$ and $\beta$ it is possible to link the WRG formulas and the FT ones. Taking $K_{6}=1 / 36$ we found that $\beta_{\mathrm{W}} \rightarrow-\beta, \gamma_{\phi} \rightarrow \gamma$ and $\bar{\gamma} \rightarrow-\gamma_{\phi^{2}}$. This mapping can be checked with the formulas for the critical exponents .

\footnotetext{
${ }^{4}$ We have recast all the formulas of these references [13, 18] to the notation of the Amit's book [7]. For $\beta(w)$ and $\gamma_{\phi}(w)$ there are no changes. The only difference is on $\bar{\gamma}_{\phi^{2}}$. The rule is: $\bar{\gamma}_{\phi^{2}}=-\gamma_{\phi^{2}}$ (REFERENCES). Here for $\gamma_{\phi^{2}}$ (REFERENCES) we mean the value of $\gamma_{\phi^{2}}$ found in 13, 18 .

${ }^{5}$ The critical exponents in FT are [7]: $\eta=\gamma_{\phi}\left(w^{*}\right)$ and $\nu=1 /\left(2-\gamma_{\phi^{2}}\left(w^{*}\right)\right)$.
} 


\begin{tabular}{|c|c|c|c|}
\hline & PERC & $m$-SG & LY-S \\
\hline \hline$\alpha$ & -1 & $-4 m$ & -1 \\
\hline$\beta$ & -2 & $1-3 m$ & -1 \\
\hline
\end{tabular}

Table 1: Values of $\alpha$ and $\beta$ for percolation (PERC), $m$-component vector spin glass $(m$-SG) and Lee-Yang singularities (LY-S).

Finally, it is possible to write a Callan-Symanzik like formula for the inverse of the susceptibility (see, for instance, reference [7])

$$
\left[\kappa \frac{\partial}{\partial \kappa}+\beta(u) \frac{\partial}{\partial u}-\eta(u)-\theta(u) \frac{\partial}{\partial t}\right] \chi_{R}^{-1}(t, u, \kappa)=0 .
$$

where $\theta(u) \equiv-\left(\bar{\gamma}_{\phi^{2}}(u)+\gamma_{\phi}(u)\right) ; \kappa$ is the momentum scale, $u$ is the dimensionless renormalized coupling and $t$ is the renormalized reduced temperature.

\subsection{Mean Field}

One subtle point in the present calculation is the presence of irrelevant dangerous variables . To understand when and how they appear we need to analyze the theory in the Mean Field framework.

The free energy is

$$
F\left(r_{0}, w_{0}\right)=\frac{r_{0}}{2} M^{2}+\frac{w_{0}}{3 !} M^{3} .
$$

If $r_{0}>0$ the only solution that minimizes the free energy is $M=0$. But if $r_{0}<0$ the solution is $M=2\left|r_{0}\right| / w_{0}$, and the free energy at this minimum is

$$
F_{\min }=-\frac{2}{3} \frac{\left|r_{0}\right|^{3}}{w_{0}^{2}}
$$

The specific heat is the second derivative of $F_{\min }$ with respect $r_{0}$ :

$$
C \propto \frac{\left|r_{0}\right|}{w_{0}^{2}}
$$

i.e. $\alpha=-1$, and $w_{0}$ is a dangerous variable [0. $w_{0}$ is dangerous because the RG prediction is that $w_{0} \rightarrow 0$ for larger blocking and it appears in the denominator in the free energy expression.

It is easy to obtain that

$$
\chi^{-1} \propto r_{0}
$$

i.e. $\gamma=1$ and thereby for this observable we find that it does not depend on $w_{0}$.

Finally we remark that in the Mean Field approximation we have $\chi\left(r_{0}\right) \propto \xi\left(r_{0}\right)^{2}$ : i.e. $\nu=1 / 2$.

\footnotetext{
${ }^{6}$ Mean Field also predicts that the specific heat vanishes in the whole paramagnetic phase.
} 


\section{Logarithmic corrections in temperature (WRG) for spin glasses}

The starting point is the usual formula for the propagator at momentum $\mathbf{k}$ :

$$
G\left(\mathbf{k}, r_{0}, w_{0}, \Lambda\right)=b^{2} \zeta(b) G(b \mathbf{k}, r(b), w(b), \Lambda)
$$

where $\zeta(b)$ is defined as

$$
\frac{\mathrm{d} \log \zeta(b)}{\mathrm{d} \log b} \equiv-\gamma(w(b))
$$

$\gamma(w)$ was defined in Eq. (9) and $\Lambda$ is the cut-off (see reference [8] for more details). Solving Eq. (17) we obtain

$$
\zeta(b) \simeq(\log b)^{m /(3(2 m-1))} .
$$

The susceptibility is nothing but the propagator at zero momentum, and so

$$
\chi\left(r_{0}, w_{0}, \Lambda\right)=b^{2} \zeta(b) \chi(r(b), w(b), \Lambda) .
$$

An equivalent approach is to start from the following formula for the singular part of the free energy $([1])$

$$
f_{\text {sing }}\left(r_{0}, w_{0}, h_{0}\right)=b^{-6} f_{\text {sing }}(r(b), w(b), h(b)),
$$

where $h(b)$ is the re-scaled magnetic field that satisfies the following recursion formula

$$
h(b)=b^{d_{h}} h_{0},
$$

with $d_{h}=(d-\gamma(w)) / 2+1$.

The susceptibility reads

$$
\left.\chi \propto \frac{\partial^{2} f_{\text {sing }}}{\partial h_{0}^{2}}\right|_{h_{0}=0},
$$

obtaining again Eq. (18).

Taking, as usual, a $b^{*}$-value such that $t\left(b^{*}\right)=1$, i.e.,

$$
b^{*} \propto t_{0}^{-1 / 2}\left(\log t_{0}\right)^{5 m /(6(2 m-1))},
$$

we obtain

$$
\chi \propto t_{0}^{-1}\left(\log t_{0}\right)^{2 m /(2 m-1)} .
$$

The correlation length verifies

$$
\xi\left(r_{0}, w_{0}, \Lambda\right)=b \xi(r(b), w(b), \Lambda)
$$

and therefore

$$
\xi \propto b^{*}=t_{0}^{-1 / 2}\left(\log t_{0}\right)^{5 m /(6(2 m-1))}
$$

We remark that the remaining factors in the above deduction (i.e. $\xi(1, w(b), \Lambda)$ and $\chi(1, w(b), \Lambda))$ do not diverge (see Eq. (16)): they are the correlation and the susceptibility 
far away of the critical point; and so computed in the Mean Field approximation. We have also found above that in the Mean Field Calculation $\xi$ and $\chi$ do not depend on $w$.

We can finally write down the formulas for the Ising spin glass $(m=1)$ :

$$
\begin{aligned}
\chi & \propto t_{0}^{-1}\left(\log t_{0}\right)^{2}, \\
\xi & \propto t_{0}^{-1 / 2}\left(\log t_{0}\right)^{5 / 6} .
\end{aligned}
$$

At this point, we can compare with the analytical result of Fisch and Harris [23] where it was found $t_{0}^{-1}\left(\log t_{0}\right)^{2}$, and so we use their result for the susceptibility as check of our calculation.

In general the susceptibility verifies $\chi=\xi^{2-\eta}$. In the $\phi^{4}$ theory, in four dimensions, $\phi_{4}^{4}$, it is clear that $\eta=0$ and $\chi=\xi^{2}$, while in $\phi^{3}$ in six dimension (which we denote as $\phi_{6}^{3}$ ), we have again that $\eta=0$ but with induced logarithmic corrections and so $\chi \neq \xi^{2}$. This fact is related with the fact that in $\phi_{4}^{4}, \zeta=$ Constant, while in the $\phi_{6}^{3}$ this does not hold.

For the specific heat we use again the expression the singular part of the free energy (Eq. (3)). The singular part of the specific heat is

$$
\left.C \propto \frac{\mathrm{d}^{2} f_{\text {sing }}}{\mathrm{d} t_{0}^{2}}\right|_{h_{0}=0} .
$$

We choose again the same $b^{*}$ as above and we can finally write

$$
C \propto t_{0}\left(\log t_{0}\right)^{-\frac{3 m+1}{2 m-1}}
$$

where we have used that in the Mean Field approximation the specific heat behaves like $C \propto 1 / w(b)^{2}$ (see Eq. (15)). In particular, fixing $m=1$, one gets

$$
C \propto t_{0}\left(\log t_{0}\right)^{-4}
$$

\section{Finite Size Scaling formulas with logarithmic correc- tions (WRG) for spin glasses}

The scaling of the singular part of the free energy in the presence of a magnetic field, $h_{0}$, is (in six dimensions) [11]

$$
f_{\text {sing }}\left(r_{0}, w_{0}, h_{0}, \frac{1}{L}\right)=b^{-6} f_{\text {sing }}\left(r(b), w(b), h(b), \frac{b}{L}\right),
$$

where we have introduced a new coupling, the system size $L$, which scales trivially with a RG transformation $(1 / L \rightarrow b / L)$. As usually the magnetic field verifies [8]:

$$
\frac{\mathrm{d} \log h(b)}{\mathrm{d} \log b}=\frac{d}{2}+1-\frac{\gamma(w)}{2} \text {. }
$$

In the asymptotic regime, the solution of (26) is

$$
h(b)=h_{0} b^{4}(\log b)^{\frac{m}{6(2 m-1)}} .
$$


Performing a RG transformation with $b=L$, we keep just one degree of freedom (see ref. [28] for more details). The free energy of this system reads

$$
f\left(r^{\prime}, w^{\prime}, h^{\prime}, L=1\right) \equiv \log \int \mathrm{d} \phi \exp \left\{-\left[\frac{r^{\prime}}{4} \phi^{2}-h^{\prime} \phi-(n-2) w^{\prime} \phi^{3}\right]\right\} .
$$

Using the standard approach [28] we re-scale the $\phi$ variable by means of $\phi^{\prime}=w^{1 / 3} \phi$. The free energy can be written as

$$
f\left(r^{\prime}, w^{\prime}, h^{\prime}, L=1\right)=\hat{f}\left(\frac{r^{\prime}}{w^{\prime 2 / 3}}, \frac{h^{\prime}}{w^{\prime 1 / 3}}\right)
$$

obtaining finally

$$
f_{\text {sing }}\left(r_{0}, w_{0}, h_{0}, \frac{1}{L}\right)=L^{-6} \hat{f}\left(\frac{r(L)}{w(L)^{2 / 3}}, \frac{h(L)}{w(L)^{1 / 3}}\right) .
$$

This formula also holds for a generic $\phi^{3}$.

We remark that $w$ is a dangerous (marginally) irrelevant variable [29, 30] and we need to do with care all the analytical steps (it is not correct to substitute $w$ for its asymptotically value, $w=0$, because the free energy depends on inverse powers of $w$ ).

To compute the thermodynamical quantities in the critical region one just need to take the appropriate derivatives of $f_{\text {sing. }}$. In order to compute the logarithmic corrections it will prove useful take into account Eqs. (6) and (25), and the following Taylor expansion (which depends on $r(L)=t(L)-36 A(0) m w(L)^{2}$ and that $t_{0}=0$ implies $\left.t(L)=0\right)$

$$
\begin{aligned}
\left.\partial_{i}^{2} \hat{f}\left(r(L) / w(L)^{2 / 3}, 0\right)\right|_{t_{0}=0} & =\partial_{i}^{2} \hat{f}\left(-36 A(0) m w(L)^{4 / 3}, 0\right) \\
& =\partial_{i}^{2} \hat{f}(0,0)+O\left(w(L)^{4 / 3}\right)
\end{aligned}
$$

where $\partial_{i}$ is the partial derivative with respect to the $i$-th argument.

The equation (29) gives us the leading correction to the scaling: it is just the term $O\left(w(L)^{4 / 3}\right)$ that modifies the scaling behavior given by $\partial_{i}^{2} \hat{f}(0,0)$. In a $m$-component spin glass we should expect correction to scaling proportional to $1 /(\log L)^{2 / 3}$.

As we are interested in the behavior with the lattice size just at the infinite volume critical temperature $\left(t_{0}=0\right)$, the susceptibility can be written as

$$
\begin{aligned}
\chi & \left.\propto \frac{\partial^{2} f_{\text {sing }}}{\partial h_{0}^{2}}\right|_{h_{0}=t_{0}=0}=\left.L^{-6}\left(\frac{\partial h(L)}{\partial h_{0}}\right)^{2} \frac{\partial^{2}}{\partial h(L)^{2}} \hat{f}\left(r(L) / w(L)^{2 / 3}, h(L) / w(L)^{1 / 3}\right)\right|_{h_{0}=t_{0}=0} \\
& \propto L^{2}(\log L)^{\frac{3 m-1}{3(2 m-1)}}\left[1+\frac{A}{(\log L)^{2 / 3}}\right]
\end{aligned}
$$

where $A$ is a constant.

At this point we can compare our prediction for the logarithmic correction for one component spin glass

$$
\chi \propto L^{2}(\log L)^{\frac{2}{3}}
$$

with which was found in numerical simulations by Wang and Young [26:

$$
\chi \propto L^{2}(\log L)^{0.64} .
$$


The agreement is very good.

The specific heat can be computed analogously

$$
\begin{aligned}
C & \left.\propto \frac{\partial^{2} f_{\text {sing }}}{\partial t_{0}^{2}}\right|_{h_{0}=t_{0}=0}=\left.L^{-6}\left(\frac{\partial r(L)}{\partial t_{0}}\right)^{2} \frac{\partial^{2}}{\partial r^{2}} \hat{f}\left(r(L) / w(L)^{2 / 3}, h(L) / w(L)^{1 / 3}\right)\right|_{h_{0}=t_{0}=0} \\
& \propto L^{-2}(\log L)^{-\frac{2(3 m+1)}{3(2 m-1)}} .
\end{aligned}
$$

At zero magnetic field, the correlation length scales as

$$
\left.\xi\left(r_{0}, w_{0}, 1 / L\right)\right|_{t_{0}=0}=\left.L \xi(r(L), w(L), 1)\right|_{t_{0}=0},
$$

where $\xi(r(L), w(L), 1)$ must be evaluated with the free energy (27). Consequently, the mass squared term is

$$
\left(\left.\xi(r, w, 1)\right|_{t_{0}=0}\right)^{-2}=\left.\frac{r(L)}{w(L)^{2 / 3}}\right|_{t_{0}=0} \propto w(L)^{4 / 3},
$$

and so

$$
\xi\left(r_{0}, u_{0}, 1 / L\right) \propto L w(L)^{-2 / 3} \propto L(\log L)^{\frac{1}{3}} .
$$

The independence of the logarithmic corrections of the correlation length on the number of components for spin glasses is similar to the $\phi_{4}^{4}$ case, where no dependences on the number of components was found in the exponent of the logarithmic corrections [10].

Finally we can also compute the shift of the apparent critical temperature. It can be defined as the temperature where the susceptibility (or specific heat) measured in a finite volume shows a maximum. Using the formula (30) for the susceptibility without imposing $t_{0}=0$ we obtain

$$
\chi \propto L^{2}(\log L)^{\frac{2 m}{3(2 m-1)}} \partial_{2}^{2} \hat{f}\left(r(L) / w(L)^{2 / 3}, 0\right) .
$$

The maximum of $\chi$ as a function of $L$ and $t$ is not just at $t_{0}=0$, but it is fixed by the condition

$$
r(L) / w(L)^{2 / 3}=\frac{t(L)-36 A(0) m w(L)^{2}}{w(L)^{2 / 3}}=x_{\max },
$$

i.e. the function $\partial_{2}^{2} \hat{f}(x, 0)$ has a maximum at $x=x_{\max }$.

As $t \propto T_{\mathrm{c}}(\infty)-T_{\mathrm{c}}(L)$, it follows that

$$
T_{\mathrm{c}}(\infty)-T_{\mathrm{c}}(L) \propto L^{-2}(\log L)^{\frac{3 m+1}{3(2 m-1)}} .
$$

\section{$5 \quad$ Percolation and Lee-Yang Singularities Formulas}

Following the procedure used in the two previous sections we can write general formulas, not just for the spin glass case as we have done in the previous part of the paper. Thus, by using the mapping between the WRG formulas $\left(\beta_{\mathrm{W}}(w), \gamma(w)\right.$ and $\left.\bar{\gamma}(w)\right)$ and the FT ones $\left(\beta(w), \gamma_{\phi}(w)\right.$ and $\left.\gamma_{\phi^{2}}(w)\right)$ we can obtain the formulas for percolation and Lee-Yang singularities. 
The starting point are the following general formulas

$$
\begin{aligned}
\frac{\mathrm{d} \log h}{\mathrm{~d} \log b} & =\frac{d+2}{2}-\frac{\gamma(\omega)}{2}, \\
\frac{\mathrm{d} w}{\mathrm{~d} \log b} & =\beta_{\mathrm{W}}(w), \\
\frac{\mathrm{d} t}{\mathrm{~d} \log b} & =(2+\bar{\gamma}(\omega)) t,
\end{aligned}
$$

and so

$$
\begin{aligned}
\int_{w_{0}}^{w(b)} \frac{\mathrm{d} w}{\beta_{\mathrm{W}}(w)} & =\log b \\
h(b) & =h_{0} b^{(d+2) / 2} \exp \left[-\frac{1}{2} \int_{w_{0}}^{w(b)} \mathrm{d} w \frac{\gamma(w)}{\beta_{\mathrm{W}}(w)}\right], \\
t(b) & =t_{0} b^{2} \exp \left[\int_{w_{0}}^{w(b)} \mathrm{d} w \frac{\bar{\gamma}(w)}{\beta_{\mathrm{W}}(w)}\right]
\end{aligned}
$$

where $t_{0} \equiv t(b=1), h_{0} \equiv h(b=1)$ and $w_{0} \equiv w(b=1)$.

We rewrite the general formula for the singular part of the free energy

$$
f_{\text {sing }}\left(r_{0}, w_{0}, h_{0}\right)=b^{-d} f_{\text {sing }}(r(b), w(b), h(b)) .
$$

We define $b^{*}$ such that $t\left(b^{*}\right)=1$, and so $b^{*}$ is a function of $t_{0}$.

Taking two derivatives with respect to the magnetic field on the singular part of the free energy we obtain the formula for the susceptibility [

$$
\chi \propto\left(b^{*}\right)^{2} \exp \left[-\int_{w_{0}}^{w\left(b^{*}\right)} \mathrm{d} w \frac{\gamma(w)}{\beta_{\mathrm{W}}(w)}\right] .
$$

This formula is valid in any dimension and theory.

The specific heat is 8

$$
C \propto\left(b^{*}\right)^{4-d} \frac{1}{w^{2}\left(b^{*}\right)} \exp \left[2 \int_{w_{0}}^{w\left(b^{*}\right)} \mathrm{d} w \frac{\bar{\gamma}(w)}{\beta_{\mathrm{W}}(w)}\right] .
$$

This formula is valid in any dimension and only for a $\phi^{3}$ theory (we have used that in a $\phi^{3}$ theory the specific heat far from the critical point behaves as $1 / w^{2}$; in a $\phi^{4}$ theory we should change the factor $1 / w^{2}$ to $\left.1 / w\right)$.

Finally the correlation length is

$$
\xi \propto b^{*}
$$

The Finite Size Scaling Formulas are

$$
\chi \propto L^{2} \frac{1}{w(L)^{2 / 3}} \exp \left[-\int_{w_{0}}^{w(L)} \mathrm{d} w \frac{\gamma(w)}{\beta_{\mathrm{W}}(w)}\right],
$$

\footnotetext{
7 In the case of percolation we identify the susceptibility with the mean cluster size [3].

8 We identify, for percolation, the specific heat with the second derivative of the singular part of the total number of clusters, $M_{0}$, with respect to the dilution [3]. In the percolation case the temperature is identified with the probability $p$, and so the reduced temperature means $p-p_{c}$.
} 


\begin{tabular}{|c|c|c|c|}
\hline & PERC & $m$-SG & LY-S \\
\hline \hline$\chi$ & $2 / 7$ & $2 m /(2 m-1)$ & $2 / 3$ \\
\hline$C$ & $2 / 7$ & $-(1+3 m) /(2 m-1)$ & $-2 / 3$ \\
\hline$\xi$ & $5 / 42$ & $5 m /(6(2 m-1))$ & $5 / 18$ \\
\hline
\end{tabular}

Table 2: Values of the exponent of the logarithmic correction, in reduced temperature, for the susceptibility, specific heat and correlation length.

for the susceptibility. The specific heat is

$$
C \propto L^{4-d} \frac{1}{w(L)^{4 / 3}} \exp \left[2 \int_{w_{0}}^{w(L)} \mathrm{d} w \frac{\bar{\gamma}(w)}{\beta_{\mathrm{W}}(w)}\right],
$$

the correlation length is given by

$$
\xi \propto L w(L)^{-2 / 3}
$$

and the shift of the critical temperature is

$$
T_{c}(L)-T_{c}(\infty) \propto L^{-2} w(L)^{2 / 3} \exp \left[-\int_{w_{0}}^{w(L)} \mathrm{d} w \frac{\bar{\gamma}(w)}{\beta_{\mathrm{W}}(w)}\right] .
$$

The FSS formulas for the specific heat, susceptibility, correlation length and shift are valid in any dimension but only for a $\phi^{3}$ theory. For $d<6$ we have $\lim _{L \rightarrow \infty} w(L)=w^{*} \neq 0$ and we get the standard (i.e. without logs corrections) Finite Size scaling formulas.

Using the mapping between $\beta_{\mathrm{W}}(w), \gamma(w)$ and $\bar{\gamma}(w)$ and $\beta(w), \gamma_{\phi}(w)$ and $\gamma_{\phi^{2}}(w)$ of section 2.2 we can write:

$$
\begin{aligned}
\chi & \propto t_{0}^{-1}\left[\log t_{0}\right]^{\frac{2 \alpha}{4 \beta-\alpha}} \\
C & \propto t_{0}\left[\log t_{0}\right]^{-\frac{6 \alpha-4 \beta}{4 \beta-\alpha}} \\
\xi & \propto t_{0}^{-1 / 2}\left[\log t_{0}\right]^{\frac{5 \alpha}{6(4 \beta-\alpha)}}
\end{aligned}
$$

In Table $⿴$ we report the exponent of the logarithm observables for percolation, spin glasses and Lee-Yang singularities.

The results $\chi \propto t_{0}^{-1}\left[\log t_{0}\right]^{2 / 7}$ and $\xi \propto t_{0}^{-1 / 2}\left[\log t_{0}\right]^{5 / 42}$ for percolation were found solving a Callan-Symanzik equation in reference [21], and therefore we use these results as check of the above calculation.

As a function of the lattice size we found the following formulas.

$$
\begin{aligned}
\chi\left(L, t_{0}=0\right) & \propto L^{2}[\log L]^{\frac{4 \beta}{3(4 \beta-\alpha)}} \\
C\left(L, t_{0}=0\right) & \propto L^{-2}[\log L]^{-\frac{12 \alpha-8 \beta}{3(4 \beta-\alpha)}} \\
\xi\left(L, t_{0}=0\right) & \propto L[\log L]^{1 / 3} \\
\Delta T_{c} & \propto L^{-1 / 2}[\log L]^{-\frac{4 \beta-6 \alpha}{3(4 \beta-\alpha)}} .
\end{aligned}
$$

In Table 3 we report the exponent of the logarithm (in lattice size) for different models.

Moreover the leading correction to the scaling, for all the models described in this paper (in general for all the models described by a generalized $\phi^{3}$ ), is proportional to $1 /(\log L)^{2 / 3}$. 


\begin{tabular}{|c|c|c|c|}
\hline & PERC & $m$-SG & LY-S \\
\hline \hline$\chi$ & $8 / 21$ & $(3 m-1) /(3(2 m-1))$ & $4 / 9$ \\
\hline$C$ & $4 / 21$ & $-2(1+3 m) /(3(2 m-1))$ & $-4 / 9$ \\
\hline$\xi$ & $1 / 3$ & $1 / 3$ & $1 / 3$ \\
\hline$\Delta T$ & $-2 / 21$ & $(1+3 m) /(3(2 m-1))$ & $2 / 9$ \\
\hline
\end{tabular}

Table 3: Values of the exponent of the logarithmic correction, in lattice size, for the susceptibility, specific heat and correlation length. We also show the logarithm factor for shift of the apparent critical temperature with the lattice size.

Finally, we can re-obtain part of the previous results (in temperature) using standard Field Theoretical techniques.

The starting point is the solution of the Callan-Symanzik like equation for the susceptibility (see Eq. (14)). The solution of this equation is [7]

$$
\chi_{R}^{-1}\left(r_{0}, w\right) \propto r_{0} \exp \left[-\int_{1}^{r_{0}}(\bar{\eta}(w(x))+\bar{\theta}(w(x))) \frac{\mathrm{d} x}{x}\right],
$$

where

$$
\begin{gathered}
\bar{\eta}(w) \equiv \frac{\gamma_{\phi}(w)}{2-\gamma_{\phi^{2}}(w)}, \\
\bar{\theta}(w) \equiv-\frac{\gamma_{\phi^{2}}(w)}{2-\gamma_{\phi^{2}}(w)},
\end{gathered}
$$

and $w(x)$ verifies

$$
\frac{\mathrm{d} w}{\mathrm{~d} \log x}=\bar{\beta}(w) \equiv \frac{\beta(w)}{2-\gamma_{\phi^{2}}(w)},
$$

with the initial condition $w(x=1)=\hat{w}_{0}$.

Using the formulas (13) of the FT approach we find

$$
\chi_{R}^{-1}\left(r_{0}\right) \propto r_{0}\left(\log r_{0}\right)^{2 \alpha /(\alpha-4 \beta)} .
$$

And we find again the same law (see Eq. (45)).

We can repeat the above calculation for $\xi^{-2}$. In this case, solving the correspondent Callan-Symanzik equation for $\xi^{-2}$, we arrive to the following formula [7]

$$
\xi^{-2}\left(r_{0}, w\right) \propto r_{0} \exp \left[-\int_{1}^{r_{0}} \bar{\theta}(w(x)) \frac{\mathrm{d} x}{x}\right] .
$$

Being the solution

$$
\xi^{-1} \propto r_{0}^{1 / 2}\left(\log r_{0}\right)^{5 \alpha /(6 \alpha-24 \beta)} .
$$

Again we have obtained the same result (see Eq. (47)). 


\section{Lee-Yang singularities and lattice animals}

In this section we will compute the singular part of the free energy for a $\phi^{3}$ theory with imaginary coupling at criticality (which describes the LY singularities [34]) as a function of the magnetic field (the results of the preceding section for this model were as a function of the reduced temperature or lattice size- in both cases in absence of magnetic field-).

Once that we have this result we will compare it with the formula found for lattice animals.

M. Fisher showed [34] a mapping between the Ising model with magnetic field in the paramagnetic phase and a $\phi^{3}$ theory with an imaginary coupling at its critical point. Following the steps described above, the first goal is to compute the Mean Field behavior. The free energy for a $\phi^{3}$ at its critical point in presence of a magnetic field $h_{0}$ is (see reference [34] for more details)

$$
F\left(r_{0}=0, w_{0}, h_{0}\right)=h_{0} M+\frac{w_{0}}{3 !} M^{3} .
$$

By computing the minimum of the free energy, we can write the free energy at this minimum

$$
F_{\min } \propto \frac{h_{0}^{3 / 2}}{w_{0}^{1 / 2}} .
$$

The next step is to write the renormalization group equation for the singular part of the free energy at the critical point

$$
f_{\text {sing }}\left(r_{0}=0, w_{0}, h_{0}\right)=b^{-6} f_{\text {sing }}(0, w(b), h(b)) .
$$

Now, $h_{0}$ is the relevant parameter and so we choose $b^{*}$ by means the relation $h\left(b^{*}\right)=1$, obtaining (by solving the first and second equations of Eqs. (36))

$$
b^{*}\left(h_{0}\right) \simeq h_{0}^{-1 / 4}\left(\log h_{0}\right)^{-1 / 72}
$$

Finally we can write the behavior of the singular part of the free energy

$$
f_{\text {sing }} \simeq\left(b^{*}\right)^{-6} \frac{h\left(b^{*}\right)^{3 / 2}}{w\left(b^{*}\right)^{1 / 2}},
$$

where we have used Eq. (53). Using $h\left(b^{*}\right)=1$, and the behavior of $w$ with $b$ we obtain

$$
f_{\text {sing }} \simeq h_{0}^{3 / 2}\left(\log h_{0}\right)^{1 / 3} .
$$

This is the behavior of the singular part of the free energy of the LY singularities in six dimensions. This formula defines the $\sigma$ exponent for the LY singularities by means: $f_{\text {sing }}=$ $h_{0}^{\sigma+1}$. Obviously we have recovered the MF result: $\sigma=1 / 2$ but modified by a logarithmic factor.

If the mapping proposed by Parisi and Sourlas holds then the behavior of the singular part of the free energy for lattice animals in eight dimension should be given by Eq. (57) (changing the magnetic field by the fugacity), but this formula is just the formula computed by Lubensky and Isaacson for the singular part of the free energy for lattice animals in eight dimensions [31]. Another test of this formula was done in reference [32] using series expansions directly on lattice animals.

The above calculation (57) supports again the correctness of the mapping between lattice animals in $d+2$ dimensions and the LY singularities in $d$ dimensions. 


\section{Conclusions}

In this paper we have computed the logarithmic corrections for a generic $\phi^{3}$ theory at its upper critical dimension both in the reduced temperature as well as in the size of the system at criticality. Moreover we have computed the leading corrections to the scaling and the shift of the apparent critical temperature.

We have distinguish the formulas to the following cases: percolation, $m$-component spin glasses and Lee-Yang singularities.

We have compared the results for the one-component spin glass with the corrections found numerically and the agreement between the theory and the simulations is very good.

Therefore we believe that the present computation of the logarithmic corrections for the one component spin glass and the agreement of these with the numerical simulations strongly support that six is the upper critical dimension for $m=1$ spin glasses.

Finally we have tested the (perturbative) mapping between lattice animals in eight dimensions and LY singularities in 6 dimensions by computing the free energy in the LY model.

\section{Acknowledgments}

J. J. R.-L. is supported by an EC HMC (ERBFMBICT950429) grant. We wish to thank H. G. Ballesteros, L. A. Fernández, V. Martín Mayor, A. Muñoz Sudupe, G. Parisi and D. Stauffer for interesting discussions. We also wish to thank the referees for pointing us useful comments and the link between LY singularities and lattice animals. 


\section{References}

[1] J. L. Cardy (ed), Finite-Size Scaling (North-Holland, Amsterdam 1988).

[2] P. Young (ed.), Spin Glasses and Random Fields (World Scientific, Singapore 1997).

[3] D. Stauffer and A. Aharony, Introduction to the Percolation Theory (Taylor and Francis,London 1994) (Revised second edition).

[4] T. D. Lee and C. N. Yang, Phys. Rev. 87, 404 (1952).

[5] G. Parisi, Field Theory, Disorder and Simulations (World Scientific, Singapore 1994).

[6] K. G. Wilson, Rev. Mod. Phys. 47, 773 (1975).

[7] D. J. Amit, Field Theory, the Renormalization Group, zinand Critical Phenomena (World Scientific, Singapore 1984) (Revised second edition). Gen

[8] M. Le Bellac, Quantum and Statistical Field Theory (Oxford Science Publications 1991).

[9] J. Zinn-Justin, Quantum Field Theory and Critical Phenomena (Oxford Science Publications 1990).

[10] E. Bré, J. Physique 43, 15 (1982).

[11] J. Salas and A. Sokal, J. Stat. Phys. 88 (1997) 567

[12] D. J. Amit, J. Phys. A: Math. . 9, 1441 (1976).

[13] O. F. de Alcantara, J. E. Kirkham and A. J. McKane, J. Phys. A: Math. Gen. 13, L247 (1980).

[14] W. L. McMillan, J. Phys. C 17, 3179 (1984); A. J. Bray and M. A. Moore, in Heidelberg Colloquium on Glassy Dynamics, edited by J. L. Hemmen and I. Morgenstern (Springer Verlag, Heidelberg, 1986), 121; D. S. Fisher and D. A. Huse, Phys. Rev. Lett. 56, 1601 (1986); Phys. Rev. B 38, 386 (1988).

[15] C. M. Newman and D. L. Stein, Phys. Rev. E. 57, 1356 (1998) and references therein.

[16] H. G. Ballesteros, L.A. Fernández, V. Martin-Mayor, A. Muñoz Sudupe and G. Parisi, Nucl. Phys. B 512, 681 (1998).

[17] D. Fisher and H. Sompolinsky, Phys. Rev. Lett., 54, 1063 (1985).

[18] O. F. de Alcantara, J. E. Kirkham and A. J. McKane, J. Phys. A: Math. Gen. 14, 2391 (1981).

[19] J. E. Green, J. Phys. A: Math. Gen. 17, L43 (1985).

[20] A. B. Harris, T. C. Lubensky and J-H. Chen, Phys. Rev. Lett. 36, 415 (1976). 
[21] J. W. Essam, D. S. Gaunt and A. J. Guttmann, J. Phys. A:Math and Gen 11, 1983 (1978).

[22] J. Adler, Y. Meir, A. Aharony and A. B. Harris, Phys. Rev. B, 41, 9183 (1990).

[23] R. Fisch and A. B. Harris. Phys. Rev. Lett. 38, 785 (1977).

[24] L. Klein et al. Phys. Rev. B 4311249 (1991).

[25] E. Luijten and W. J. Blöte, Phys. Rev. Lett. 76, 1557 (1996); Erratum 76, 3662 (1996).

[26] J. Wang and A. P. Young, J. Phys. A: Math. Gen. 26, 1063 (1993).

[27] H. G. Ballesteros, L.A. Fernández, V. Martin-Mayor, A. Muñoz Sudupe, G. Parisi and J. J. Ruiz-Lorenzo, Phys. Lett. B 400, 346 (1997).

[28] E. Luijten and W. J. Blöte, Phys. Rev. Lett. 76, 1557 (1996); Erratum 76 (1996) 3662.

[29] A. C. D. van Enter, R. Fernández and A. D. Sokal, J. Stat. Phys. 72 (1994) 879.

[30] M. E. Fisher, in Renormalization Group in Critical Phenomena and Quantum Field Theory: Proceedings of a conference. Temple University ( Philadelphia, 1974).

[31] T. C. Lubensky and J. Isaacson, Phys. Rev. Lett. 41, 829 (1978); 42, 410(E) (1979); Phys. Rev. A 20, 2130 (1979).

[32] J. Adler et al., Phys. Rev. B 38, 4941 (1988).

[33] G. Parisi and N. Sourlas, Phys. Rev. Lett. 46, 871 (1981).

[34] M. E. Fisher, Phys. Rev. Lett. 40, 1610 (1978).

[35] A. B. Harris and T. C. Lubensky, Phys. Rev. B 24, 2656 (1981). 